\title{
A Facile Synthesis of AuAg Alloy Nanoparticles Using a Chemical Reaction Induced by Sputter Deposition of Metal onto Ionic Liquids
}

\author{
Toshimasa Suzuki, ${ }^{a}$ Ken-ichi Okazaki, ${ }^{a}$ Tomonori Kiyama, ${ }^{a}$ Susumu Kuwabata, ,,c \\ and Tsukasa ToRIMOTO ${ }^{\mathrm{a}, \mathrm{c}, *}$
}

\author{
a Graduate School of Engineering, Nagoya University (Furo-cho, Chikusa-ku, Nagoya 464-8603, Japan) \\ b Graduate School of Engineering, Osaka University (Suita, Osaka 565-0871, Japan) \\ c Japan Science and Technology Agency, CREST (Kawaguchi, Saitama 332-0012, Japan)
}

\section{Received February 23, 2009 ; Accepted June 19, 2009}

\begin{abstract}
Sputter deposition of silver (Ag) onto an ionic liquid (IL) produced Ag nanoparticles whose size was varied greatly dependent on the sputtering conditions. Change in discharge current from 10 to $40 \mathrm{~mA}$ increased Ag particle size from 5.7 to $11 \mathrm{~nm}$, though prolongation of sputtering time simply caused a higher concentration of $\mathrm{Ag}$ nanoparticles in IL without change in their size. Sputter deposition of Ag onto IL solutions containing $\mathrm{HAuCl}_{4}$ resulted in the appearance of a single surface plasmon resonance peak in the absorption spectra of the resulting solution, and their peak position was red-shifted with an increase in the concentration of $\mathrm{HAuCl}_{4}$. The obtained results clearly indicated that $\mathrm{Ag}$ metal species sputter-deposited in IL can reduce $\mathrm{HAuCl}_{4}$ to give $\mathrm{AuAg}$ alloy nanoparticles and that their chemical composition varies depending on the initial concentration of $\mathrm{HAuCl}_{4}$.
\end{abstract}

Key Words : Ionic Liquids, Nanoparticles, Sputter Deposition, Alloy, Surface Plasmon Resonance

\section{Introduction}

Ionic liquids (ILs) have been promising solvents for various reactions such as organic ${ }^{1,2)}$ and inorganic ${ }^{3,4)}$ syntheses and electrochemical reactions.,5) Preparation of metal nanoparticles has attracted much attention because they could catalyze unique reactions in ILs that did not occur in conventional organic or aqueous solvents. Several strategies have been developed for the preparation of metal particles, such as $\mathrm{Pt},{ }^{7)} \mathrm{Rh},{ }^{8)} \mathrm{Ir},{ }^{8)}$ and $\mathrm{Au},{ }^{9)}$ uniformly dispersed in ILs. Recently, we have developed a clean method to prepare metal nanoparticles in ILs without any additives, such as reducing agents and/or stabilizing agents. The extremely low vapor pressure of ILs enabled sputter deposition of metal species onto ILs under vacuum, resulting in the formation of highly dispersed metal nanoparticles, such as $\mathrm{Au}, \mathrm{Ag}$ and AuAg, having sizes of $2 \sim 5 \mathrm{~nm} \cdot{ }^{10,11)}$ Furthermore, it has been reported that injection of low-energy electrons into ILs induced the reduction of metal ions, such as $\mathrm{Ag}^{+12,13)}$ and $\mathrm{AuCl}_{4}{ }^{-14)}$ to produce corresponding metal particles. If metal nanoparticles having an oxidation potential were sputter-deposited in ILs containing another metal ions having more positive reduction potential, the latter metal ions could be reduced to form nanoparticles. However, such an attempt has never been made.

In this study, we prepared Ag nanoparticles having various sizes by sputter-deposition onto an IL with different conditions. Furthermore, Ag-sputtering was made onto IL solutions containing $\mathrm{HAuCl}_{4}$ to produce $\mathrm{AuAg}$ alloy particles.

\section{Experimental}

An IL of 1-butyl-3-methylimidazolium hexafluorophos-
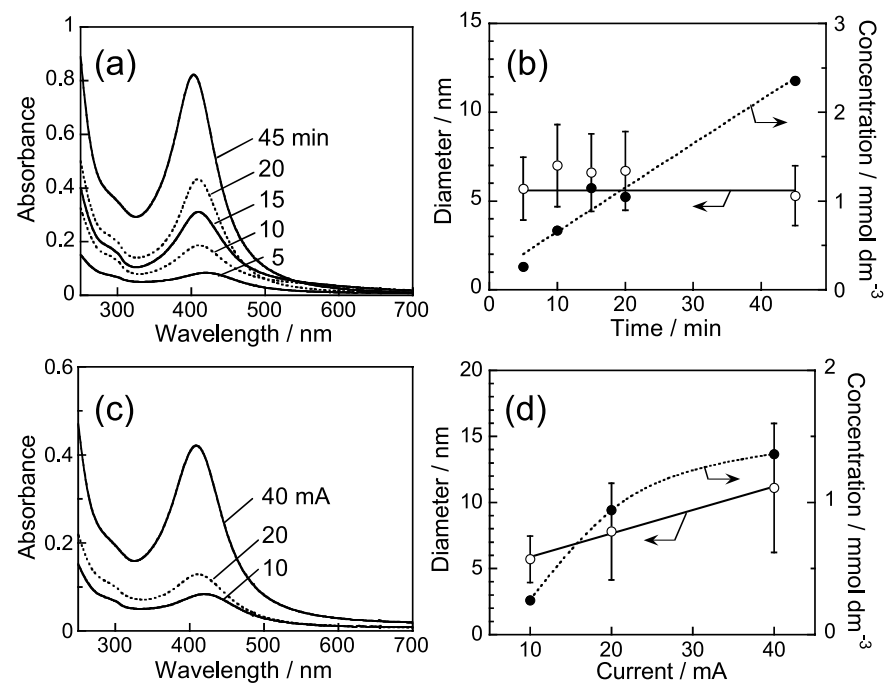

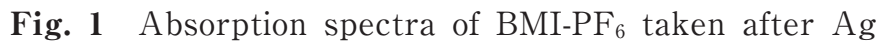
sputter deposition (a, c) and the dependence of average size (open circles) and concentration (solid circles) of the thusobtained $\mathrm{Ag}$ nanoparticles on sputtering conditions (b, d). The discharge current of $10 \mathrm{~mA}$ was used for $\mathrm{Ag}$ sputtering $(\mathrm{a}, \mathrm{b})$, or the sputtering time was fixed to $5 \mathrm{~min}$ (c, d). The optical length of a quartz cuvette used was 1.0 $\mathrm{mm}$. The numbers in figures represent the sputtering time (a) or the discharge current (c). The error bars in figure b and $\mathrm{d}$ indicate the size distribution.

phate $\left(\mathrm{BMI}_{-} \mathrm{PF}_{6}\right)$ was purchased from Kanto Chemical Co., Inc. A portion of $\mathrm{HAuCl}_{4}$ ethanol solution was added

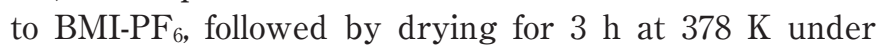
vacuum. A $0.60 \mathrm{~cm}^{3}$ portion of thus-obtained solution was 


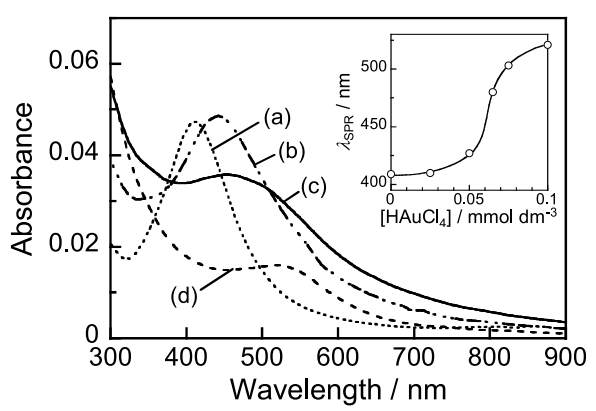

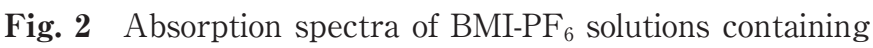
0 (a), 50 (b), 65 (c), and $100 \mu \mathrm{mol} \mathrm{dm}{ }^{-3}$ (d) $\mathrm{HAuCl}_{4}$ taken after sputter deposition of Ag. The optical length of a quartz cuvette used was $1.0 \mathrm{~mm}$. The SPR peak wavelengths are plotted as a function of the initial concentration of $\mathrm{HAuCl}_{4}$ (inset). Sputter deposition of $\mathrm{Ag}$ onto ILs was carried out for $5 \mathrm{~min}$ with a discharge current of $10 \mathrm{~mA}$.

spread on a glass plate $\left(10 \mathrm{~cm}^{2}\right)$ that was horizontally set in a DC sputtering apparatus (Sanyu Electron Co., Ltd, SC-701HMCII). It was located at a distance of $85 \mathrm{~mm}$ from the silver (99.99\% in purity) foil target. Sputter deposition of $\mathrm{Ag}$ in ILs was carried out under an argon pressure $(5 \mathrm{~Pa})$ at room temperature. The concentration of $\mathrm{Ag}$ deposited in IL was determined by using X-ray fluorescence spectrometer (XRF; Rigaku EDXL300). A $0.2 \mathrm{~cm}^{3}$ portion of $\mathrm{Ag}$ particle IL solution was mixed with $1.0 \mathrm{~cm}^{3}$ methanol solution containing 1,6-hexanedithiol $\left(0.05 \mathrm{~cm}^{3}\right)$, followed by the centrifugation. Thus-obtained precipitates of $\mathrm{Ag}$ particles were dissolved in a $\mathrm{HNO}_{3}$ aqueous solution and then subjected to the XRF analysis.

For the preparation of TEM and XPS samples, metal nanoparticles dispersed in ILs were isolated by precipitation with addition of a small amount of acetonitrile to $\mathrm{BMI}^{-\mathrm{PF}_{6}}$ solution, washed with acetonitrile several times, and dispersed in acetonitrile. The structure and size distribution of nanoparticles were examined using a HITACHI H-7650 transmission electron microscope (TEM) with an acceleration voltage of $100 \mathrm{kV}$. TEM samples were prepared by dropping the resulting suspension onto a copper TEM grid with amorphous carbon overlayers, followed by removal of the excess amount of solution with a filter paper. Elemental composition of isolated AuAg alloy particles was examined with X-ray photoelectron spectroscopy (XPS; JEOL JPS-9000MC), by considering peak areas of each band in XPS spectra and their corresponding relative sensitivity factor.

\section{Results and Discussion}

Figure 1 (a) shows changes in the absorption spectra of $\mathrm{BMI}^{-\mathrm{PF}_{6}}$ by sputter deposition of $\mathrm{Ag}$ with various sputtering times. A sharp absorption peak at ca. $420 \mathrm{~nm}$ that developed with elapse of sputtering time was assigned to the surface plasmon resonance (SPR) peak of $\mathrm{Ag}$ nanoparticles. The concentration of $\mathrm{Ag}$ linearly

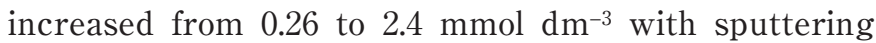
time from 5 to 45 min (Fig. 1 (b)). TEM observation revealed that the spherical $\mathrm{Ag}$ nanoparticles with a size of ca. $5.7 \mathrm{~nm}$ were formed regardless of sputtering time. Similar behavior was observed for Au sputter deposition (a)
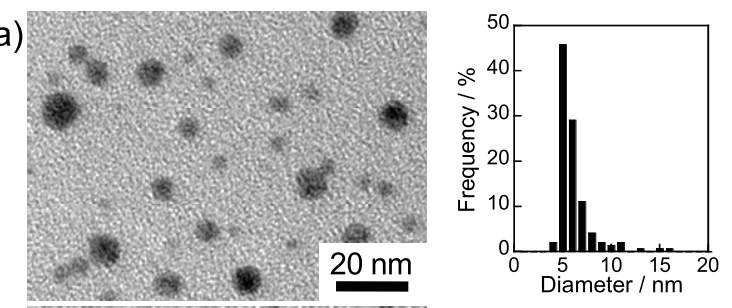

(b)

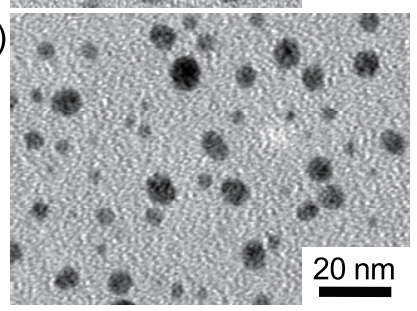

(c)
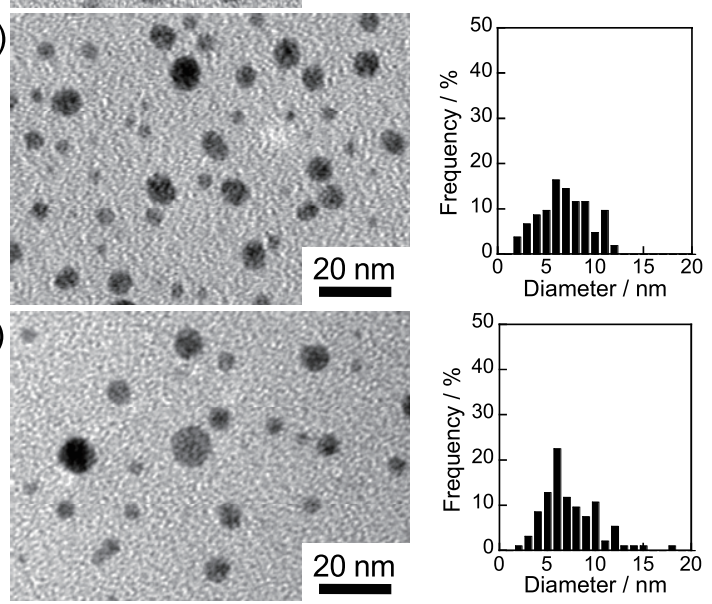

Fig. 3 TEM images of Ag nanoparticles sputter-deposited in pure $\mathrm{BMI}^{-\mathrm{PF}_{6}}$ (a) and $\mathrm{AuAg}$ nanoparticles prepared in BMI-PF 6 containing 65 (b) and 100 (c) $\mu \mathrm{mol} \mathrm{dm} \mathrm{dm}^{-3} \mathrm{HAuCl}_{4}$. Size distributions are also shown on the right side of the corresponding TEM images.

in our previous study. ${ }^{11)}$ On the other hand, as shown in Fig. 1 (c), the SPR peak intensity of Ag particles increased with an increase in discharge current from 10 to $40 \mathrm{~mA}$, resulting in an increase in the concentration of Ag from 0.26 to $1.4 \mathrm{mmol} \mathrm{dm}^{-3}$. The size of nanoparticles formed also increased from 5.7 to $11 \mathrm{~nm}$ with an increase in discharge current from 10 to $40 \mathrm{~mA}$. In sputter deposition, bombardment of energetic gaseous ions on the $\mathrm{Ag}$ foil surface causes physical ejection of surface atoms and/or clusters, which are injected into the IL solution. The density of these Ag species at the solution surface became high enough to coalesce with each other, the degree being remarkable with high discharge current. The coalescence of sputtered species proceeds until Ag nanoparticles have been stabilized by the adsorption of ions of the IL.

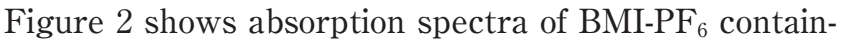
ing $\mathrm{HAuCl}_{4}$ after sputter deposition of Ag. A single SPR peak is observed in each spectrum. The peak shape became broad and then the peak wavelength $\left(\lambda_{\mathrm{SPR}}\right)$ was red-shifted with an increase in $\mathrm{HAuCl}_{4}$ concentration (inset of Fig. 2). It has been reported by $\mathrm{Xia}$ and coworkers that $\mathrm{AuCl}_{4}{ }^{-}$ions in aqueous solutions were reduced by oxidative dissolution of $\mathrm{Ag}$ metal, according to eq. 1, resulting in AuAg alloy formation of particles or hollow shells. ${ }^{15,16)}$

$$
\mathrm{AuCl}_{4}^{-}+3 \mathrm{Ag}=\mathrm{Au}+3 \mathrm{AgCl}+\mathrm{Cl}^{-}
$$

In this case also, it seems reasonable that $\mathrm{AuAg}$ alloy particles in $\mathrm{BMI}_{-} \mathrm{PF}_{6}$ were formed by the reduction of $\mathrm{HAuCl}_{4}$ with sputter-deposited $\mathrm{Ag}$ species because it has been reported that $\mathrm{AuAg}$ alloy nanoparticles exhibited a single SPR peak in absorption spectra whose wavelength 
was linearly red-shifted with an increase in the fraction of $\mathrm{Au}$ atoms in the particles. ${ }^{17)}$ It was confirmed by TEM and XPS that the chemical composition of AuAg alloy nanoparticles was changed by changing the concentration of $\mathrm{HAuCl}_{4}$ in $\mathrm{BMI}^{-} \mathrm{PF}_{6}$.

Alloy particles were isolated by the addition of acetonitrile to IL solutions. TEM observation of these particles revealed that solid spherical nanoparticles were formed in $\mathrm{BMI}^{-\mathrm{PF}_{6}}$ solutions by sputter deposition of $\mathrm{Ag}$, regardless of the concentration of $\mathrm{HAuCl}_{4}$, as shown in Fig. 3. The size determined from TEM measurements increased slightly with an increase in the initial concentration of $\mathrm{HAuCl}_{4}$ : the $\mathrm{Ag}$ nanoparticles sputter-deposited in pure BMI-PF 6 had an average diameter $\left(d_{\mathrm{av}}\right)$ of $5.7 \mathrm{~nm}$ with a standard deviation $(\sigma)$ of $1.8 \mathrm{~nm}$, while sputter deposition onto solutions containing 65 and $100 \mu \mathrm{mol} \mathrm{dm}{ }^{-3}$ $\mathrm{HAuCl}_{4}$ produced particles having $d_{\mathrm{av}}(\sigma)$ of $6.4(2.6)$ and $6.9(2.9) \mathrm{nm}$, respectively. A little larger values of $d_{\mathrm{av}}$ and $\sigma$ for AuAg alloy nanoparticles than those for Ag nanoparticles is due probably to progress of some aggregation during $\mathrm{Ag}$ oxidation and $\mathrm{Au}$ deposition reactions. However, the prepared AuAg nanoparticles were stable for several days without change in their absorption spectra.

XPS spectra of $\mathrm{AuAg}$ particles isolated from $\mathrm{BMI}^{\mathrm{P} \mathrm{PF}_{6}}$ containing $65 \mu \mathrm{mol} \mathrm{dm}{ }^{-3} \mathrm{HAuCl}_{4}$ showed that the binding energies of $\mathrm{Au} 4 \mathrm{f}_{7 / 2}$ and $\mathrm{Au} 4 \mathrm{f}_{5 / 2}$ appeared at 83.1 and $86.8 \mathrm{eV}$ and those of $\mathrm{Ag} 3 \mathrm{~d}_{5 / 2}$ and $\mathrm{Ag} 3 \mathrm{~d}_{3 / 2}$ appeared at 367.3 and $373.3 \mathrm{eV}$, respectively. The energies for gold and silver were in good agreement with the literature values reported for AuAg alloy particles. ${ }^{18)}$ Since no signals assigned to $\mathrm{Cl}$ atoms were detected, $\mathrm{AgCl}$ particles were not contained in the particles isolated from IL solutions. These facts suggest that $\mathrm{AgCl}$ particles are extremely small even if they are formed according to eq. 1 , and then the simple addition of acetonitrile to IL solutions can not cause the precipitation of $\mathrm{AgCl}$ under the conditions used in the present study. The present sputtering conditions must give $260 \mu \mathrm{mol} \mathrm{dm}{ }^{-3} \mathrm{Ag}$ concentration in the $\mathrm{BMI}^{-\mathrm{PF}_{6}}$ solutions. Therefore, according to eq. 1 , alloy nanoparticles formed in solutions containing $\mathrm{HAuCl}_{4}$ of 65 and $100 \mu \mathrm{mol} \mathrm{dm}{ }^{-3}$ are expected to form $\mathrm{AuAg}$ alloy with $\mathrm{Au} / \mathrm{Ag}$ ratios of $0.50 / 0.50$ and $1.00 / 0$, respectively. However, XPS analyses revealed the ratios of $0.34 / 0.66$ and $0.90 / 0.10$, respectively. These facts suggest that all sputtered $\mathrm{Ag}$ atoms did not react with $\mathrm{HAuCl}_{4}$ in the solutions. It is likely that the formation of $\mathrm{Au}$-rich alloy shells retards the oxidative dissolution of Ag nanoparticle cores.

\section{Conclusion}

The size of Ag nanoparticles prepared by sputterdeposition of $\mathrm{Ag}$ onto $\mathrm{BMI}^{-\mathrm{PF}_{6}}$ and their concentration varied depending on sputtering conditions, such as magnitude of discharge current. Sputtered Ag species caused reduction of $\mathrm{HAuCl}_{4}$ in the IL, resulting in the formation of $\mathrm{AuAg}$ alloy nanoparticles. The chemical composition and optical property of the alloy nanoparticles were easily controlled by changing the concentration of $\mathrm{HAuCl}_{4}$. Although only AuAg alloy was prepared in the present study, various metal and alloy nanoparticles can be prepared in ILs by appropriately selecting the metal to be sputter-deposited on ionic liquids to reduce different metal ions. Furthermore, since highly reactive metals, such as $\mathrm{Zn}$ and $\mathrm{Li}$, are in principle vacuum-deposited onto ILs to form nanoparticles, this technique will be useful for the development of novel chemical reactions in ILs under vacuum using ultrafine metal nanoparticles as reagents.

\section{Acknowledgement}

This work was supported by a Grant-in-Aid for Scientific Research on Priority Area "Strong PhotonsMolecules Coupling Fields (No. 470)" (No. 19049009) and "Science of Ionic Liquids (No.452)" (No. 20031012) from the Ministry of Education, Culture, Sports, Science and Technology of Japan. T.S. expresses his appreciation to The Global COE (Center Of Excellence) Program "Elucidation and Design of Materials and Molecular Functions" of Nagoya University.

\section{References}

1) A. C. Cole, J. L. Jensen, I. Ntai, K. L. T. Tran, K. J. Weaver, D. C. Forbes, and J. H. Davis, J. Am. Chem. Soc., 124, 5962 (2002).

2) Y. L. Gu, C. Ogawa, J. Kobayashi, Y. Mori, and S. Kobayashi, Angew. Chem. Int. Edit., 45, 7217 (2006).

3) M. A. Gelesky, A. P. Umpierre, G. Machado, R. R. B. Correia, W. C. Magno, J. Morais, G. Ebeling, and J. Dupont, J. Am. Chem. Soc., 127, 4588 (2005).

4) Y. Wang and H. Yang, Chem. Commun., 2006, 2545.

5) B. M. Quinn, Z. F. Ding, R. Moulton, and A. J. Bard, Langmuir, 18, 1734 (2002).

6) F. Endres, M. Bukowski, R. Hempelmann, and H. Natter, Angew. Chem. Int. Edit., 42, 3428 (2003).

7) C. W. Scheeren, G. Machado, S. R. Teixeira, J. Morais, J. B. Domingos, and J. Dupont, J. Phys. Chem. B, 110, 13011 (2006).

8) G. S. Fonseca, A. P. Umpierre, P. F. P. Fichtner, S. R. Teixeira, and J. Dupont, Chem. Eur. J., 9, 3263 (2003).

9) H. Itoh, K. Naka, and Y. Chujo, J. Am. Chem. Soc., 126, 3026 (2004).

10) T. Torimoto, K. Okazaki, T. Kiyama, K. Hirahara, N. Tanaka, and S. Kuwabata, Appl. Phys. Lett., 89, 243117 (2006).

11) K. Okazaki, T. Kiyama, K. Hirahara, N. Tanaka, S. Kuwabata, and T. Torimoto, Chem. Commun., 2008, 691.

12) S. A. Meiss, M. Rohnke, L. Kienle, S. Z. El Abedin, F. Endres, and J. Janek, ChemPhysChem, 8, 50 (2007).

13) S. Z. El Abedin, M. Polleth, S. A. Meiss, J. Janek, and F. Endres, Green Chem., 9, 549 (2007).

14) A. Imanishi, M. Tamura, and S. Kuwabata, Chem. Commun., 2009, 1775.

15) Y. G. Sun, B. Wiley, Z. Y. Li, and Y. N. Xia, J. Am. Chem. Soc., 126, 9399 (2004).

16) X. M. Lu, H. Y. Tuan, J. Y. Chen, Z. Y. Li, B. A. Korgel, and Y. N. Xia, J. Am. Chem. Soc., 129, 1733 (2007).

17) S. Link, Z. L. Wang, and M. A. El-Sayed, J. Phys. Chem. $B, \mathbf{1 0 3}, 3529$ (1999).

18) R. E. Watson, J. Hudis, and M. L. Perlman, Phys. Rev. B, 4, 4139 (1971). 